\title{
Individual layer fabrication (ILF): a novel approach to additive manufacturing by the use of wood
}

\author{
Klaudius Henke $^{1} \odot \cdot$ Daniel Talke $^{1} \cdot$ Frauke Bunzel $^{2} \cdot$ Birger Buschmann $^{1} \cdot$ Carsten Asshoff $^{2}$
}

Received: 21 October 2020 / Accepted: 28 November 2020 / Published online: 28 January 2021

(c) The Author(s) 2021

\begin{abstract}
A novel process named 'individual layer fabrication (ILF)' is presented, in which objects are built up by laminating individually contoured wood-based panels. However, contrary to the well-known process of 'laminated object manufacturing (LOM)', in ILF, the individual panels are not shaped by a subtractive process but additively by selective binding of wooden particles. The particles are spread as a thin layer onto a built platform. A liquid adhesive is then applied only to those areas where the contoured panel is to be generated. As each layer is fabricated individually, the ILF process allows the application of mechanical pressure. Thereby, compared to other additive manufacturing techniques, the necessary amount of binder can be significantly reduced and mechanical properties comparable to particle boards can be achieved.
\end{abstract}

\section{Introduction}

The use of wood in additive manufacturing opens up the chance to employ a renewable raw material in this rapidly developing manufacturing technology. Furthermore, next to the ecological significance, it can help enhance material properties and reduce material costs. For these reasons, using wood in additive manufacturing has gained high attention in recent years.

Fabricating objects from wood-based materials by additive manufacturing can be realized in many different ways. This includes deposition processes, where solids are built up by depositing material (e.g., fused filament fabrication or paste extrusion), as well as selective solidification processes, where solids are generated by selectively solidifying parts of a larger volume of formable material (e.g., selective laser sintering or selective binding).

In fused filament fabrication (FFF), thermoplastic material is melted and then deposited in thin strands. Standard consumables are acrylonitrile butadiene styrene (ABS) and

Klaudius Henke

henke@tum.de

1 Chair of Timber Structures and Building Construction, Technical University of Munich, Arcisstraße 21, 80333 München, Germany

2 Fraunhofer Institute for Wood Research, Wilhelm-Klauditz-Institut (WKI), Bienroder Weg 54E, 38108 Braunschweig, Germany polylactic acid (PLA), mostly in the form of a filament but also as pellets. The use of wood in FFF is realized by adding a wood-based filler to a thermoplastic matrix (commonly PLA). There are several commercial wood-filled filaments for FFF available on the market (Lamm et al. 2020).

Another group of deposition process is based on the extrusion of a paste consisting of wood particles and a fluid binder. The use of different binders like adhesives (Kariž et al. 2016), methylcellulose (Rosenthal et al. 2018) and cement (Henke et al. 2016) has been reported.

Composites of wood particles and thermoplastic polymers can also be processed by powder bed fusion (PBF) (Jiang et al. 2010). In the PBF process, thermoplastic powder is selectively melted by a laser, thus fusing the particles to form the desired solid.

Finally, wood can also be used in additive manufacturing by selective binding of wood-based bulk material. Various binders, for example methylcellulose, gypsum or cement, have been investigated (Henke and Treml 2013).

All these processes have in common that, unlike in the production of conventional wood-based panels, no mechanical pressure is applied. The consequence is that the amount of binder in additively manufactured wood-composite parts is comparatively high and that the mechanical properties are primarily determined by the binder rather than by the wood. This fact led to the search of an additive manufacturing process which would allow the application of mechanical pressure. In the presented process of individual layer fabrication (ILF), this becomes possible as each layer is fabricated 
individually before the resulting contoured panels are laminated to form the desired solid object.

\section{Materials and methods}

In the ILF process, three-dimensional objects are built up by laminating individually contoured wood-based panels. However, contrary to the process known as 'laminated object manufacturing (LOM)' (Feygin and Hsieh 1991), in ILF, the individual panels are not shaped by a subtractive process but additively by selective binding of wooden particles. As each layer is fabricated individually, the ILF process allows the application of mechanical pressure.

The process consists of a series of subsequent working steps (Fig. 1, top). It can be organized as a continuous operation, for example by the use of a conveyor belt (g). Particles are spread in a thin layer by a scattering device (a). A print head applies liquid adhesive locally, limited to those areas of the bulk that are intended to be bound (b). Mechanical pressure is then applied with a press (c), completing the fabrication of the individual layer. Finally, the unbound bulk is removed (f) and the completed contoured panels (d) are transferred, stacked and laminated to form the desired object (e). Optionally, the process can be augmented by further working steps, for example smoothing and compacting of the bulk or subtractive contouring of the panels. As in all additive manufacturing processes, the physical fabrication process is preceded by a digital process in which the shape of each single layer is generated, typically by slicing a threedimensional CAD model.

To explore the feasibility of ILF, preliminary experiments were conducted (Fig. 1, bottom). Layers of wood chips (spruce, particle size $0.8-1.1 \mathrm{~mm}$, layer thickness $2 \mathrm{~mm}$ ) were dispensed by sieving and flattened with a roller (Fig. 1, bottom, a). Adhesive (synthetic resin on the basis of polyvinyl acetate) was pumped with a progressive cavity pump to a nozzle, that was guided by a CNC-portal to apply the fluid to those regions of the layer intended to be bound (Fig. 1, bottom, b). After spreading a second layer (again $2 \mathrm{~mm}$ ) of wood chips, the $4 \mathrm{~mm}$ thick layer was pressed with a handoperated hydraulic workshop press (Fig. 1, bottom, c). After removing the unbound particles, the contoured panel (layer thickness after pressing: $2 \mathrm{~mm}$ ) could be retrieved (Fig. 1, bottom, d). Finally, the panels were laminated to form the desired object, in this case a truncated cone (base diameter $=200 \mathrm{~mm}$, upper diameter $=100 \mathrm{~mm}$, height $=100 \mathrm{~mm}$ ) (Fig. 1, bottom, e).
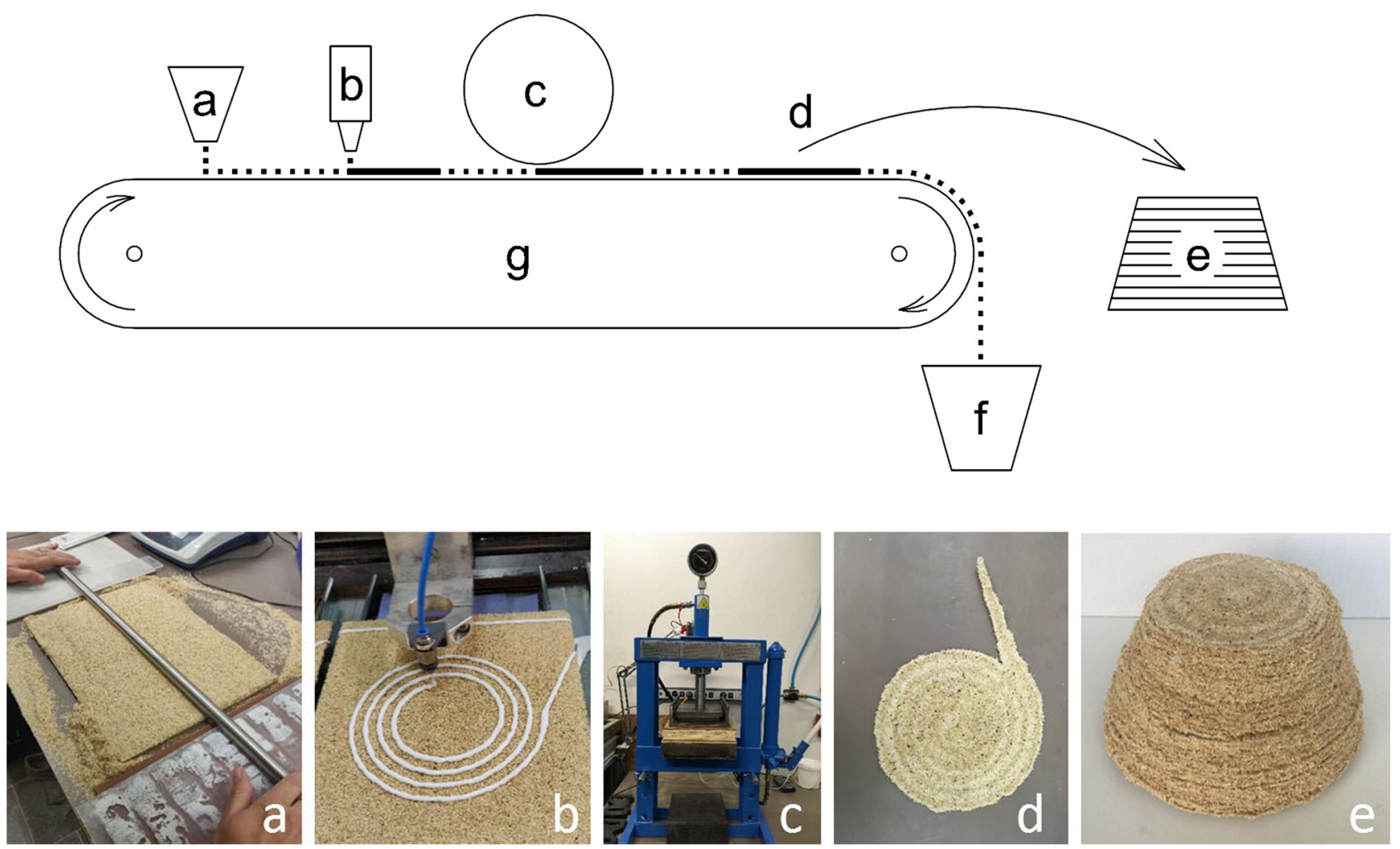

Fig. 1 Top - Basic principle of the process of individual layer fabrication (ILF) (picture: K. Henke), Bottom---Preliminary experiments (pictures: M. Anzinger), Common caption for top and bottom---dis- pensing of bulk (a), fluid application (b), pressing (c), contoured panel (d), solid object formed by laminated panels (e), removal of unbound material $(\mathbf{f})$, conveying of work pieces $(\mathbf{g})$ 


\section{Results and discussion}

The ILF process was executed by hand with simple equipment to obtain a proof of concept. The first orienting experiments delivered valuable insight into this novel process. They showed that the additive manufacturing of individual layers by selective binding of wooden particles with an adhesive is feasible and that applying mechanical pressure can be integrated into this process. The individual panels showed good bonding and an acceptable degree of shape accuracy. By laminating a series of panels, a solid object of the intended shape could be created.

Compared to most other additive manufacturing processes, the proposed solution allows the application of mechanical pressure. One conceivable way of achieving this would be to apply pressure to the three-dimensional object as a whole while building it up layer by layer. However, this may be problematic in areas of overhangs and bridges as the necessary counter pressure can be insufficient. Furthermore, issues of instability may arise in the case of certain geometries. Both issues would lead to a considerable limitation of design freedom. This can be countered by fabricating the layers individually, as is proposed here. In doing so, the pressure can be precisely controlled according to the desired density and it is ensured, that the compression is constant across the entire layer, independent of the geometry of the final three-dimensional object. Furthermore, unlike in other particle-based processes, unfilled cavities are feasible by fabricating the layers individually, which is of particular interest, when it comes to lightweight structures and functionalization of building elements.

The experiments revealed some main challenges that have to be addressed in the further development of the process. One major challenge lies in the intrusion of the adhesive into the bulk. To obtain a strong binding of the particles and a precise shape of the panels, controlling adhesive penetration into the bulk in vertical direction as well as its spreading in horizontal direction are essential. The properties of the adhesive and those of the bulk material have to be matched so that the fluid penetrates the bulk well but without oversaturating it. Furthermore, it has to be taken into account that the contour fidelity is also affected by the subsequent pressing of the layer. However, should the contour fidelity prove to be insufficient for certain applications, an additional subtractive work step (e.g., laser cutting) following the pressing can be implemented.

Another challenge concerns the stacking of the individual panels. The preliminary tests showed that the process of precisely stacking the panels and keeping them in place during laminating is not trivial. In addition, depending on the geometry of the object, issues of stability may arise during this phase. To cope with these questions, a variant of the ILF process is being explored in which the complete layer, consisting of bound and unbound particles, is passed on for stacking after pressing. In doing so, the stacking of layers with identical outlines simplifies the precise placement. In addition, the unbound material helps to support overhanging parts of the structure, thus further increasing the range of geometric freedom. Preliminary tests have shown that after pressing, the areas of unbound particles had developed sufficient stability to be able to handle them as a whole.

\section{Conclusion}

A novel process of additive manufacturing by the use of wood is presented. The process allows the application of mechanical pressure with the aim of reducing the amount of adhesive and enhancing the mechanical properties. In preliminary experiments, the feasibility of the process was proven. Challenges concerning the intrusion of adhesive into the bulk and the stacking of individual panels have been identified.

Currently, the intrusion of different adhesives (e.g., ureaformaldehyde resin (UF) or 4,4'-diphenylmethane diisocyanate (MDI)) into the bulk (e.g. ,wood flour, wood chips of different sizes) is examined in detail. Parallel to that, alternative valve systems (e.g., progressive cavity pumps, electromagnetic valves or piezoelectric drop-on-demand print heads) are being tested in regard to their suitability for these adhesives. The spreading of the bulk will be executed by a scattering machine with needle-roller and brush-off device. For the pressing of the layers, different mechanical hot presses are now available.

In the current project, the ILF process will be automated and upscaled gradually. Through the identification or new formulation of suitable materials (adhesives and bulk) and careful choosing or designing of the tools for each work step, the process will be optimized in respect to material properties, shape accuracy, geometric freedom, process speed and reliability.

Acknowledgements The research presented in this paper is being conducted within the project, Structural Timber by Individual Layer Fabrication (ILF)'. The project is part of the collaborate research center 'Additive Manufacturing in Construction - The Challenge of Large Scale', funded by the Deutsche Forschungsgemeinschaft (DFG, German Research Foundation) - project number 414265976 - TRR 277.

Funding Open Access funding enabled and organized by Projekt DEAL. 


\section{Compliance with ethical standards}

Conflict of interest The authors have no conflicts of interest to declare that are relevant to the content of this article.

Open Access This article is licensed under a Creative Commons Attribution 4.0 International License, which permits use, sharing, adaptation, distribution and reproduction in any medium or format, as long as you give appropriate credit to the original author(s) and the source, provide a link to the Creative Commons licence, and indicate if changes were made. The images or other third party material in this article are included in the article's Creative Commons licence, unless indicated otherwise in a credit line to the material. If material is not included in the article's Creative Commons licence and your intended use is not permitted by statutory regulation or exceeds the permitted use, you will need to obtain permission directly from the copyright holder. To view a copy of this licence, visit http://creativecommons.org/licenses/by/4.0/.

\section{References}

Feygin M, Hsieh B (1991) Laminated object manufacturing (LOM): a simpler process. In: Proceedings of the 1991 international solid freeform fabrication symposium. The University of Texas

Henke K, Treml S (2013) Wood based bulk material in 3D printing processes for applications in construction. Eur J Wood Prod 71(1):139-141. https://doi.org/10.1007/s00107-012-0658-Z
Henke K, Talke D, Winter W (2016) Additive manufacturing of building elements by extrusion of wood concrete. In: Proceedings of the world conference on timber engineering (WCTE). Vienna, Austria

Jiang KY, Guo YL, Zeng WL, Xin ZS (2010) The Preparation of WPC for SLS Rapid Prototyping and Manufacturing. Adv Mat Res 113-116:1722-1725. https://doi.org/10.4028/www.scientific .net/AMR.113-116.1722

Kariž M, Sernek M, Kuzman M (2016) Use of wood powder and adhesive as a mixture for 3D printing. Eur J Wood Prod 74(1):123126. https://doi.org/10.1007/s00107-015-0987-9

Lamm ME, Wang L, Kishore V, Tekinalp H, Kunc V, Wang J, Gardner DJ, Ozcan S (2020) Material extrusion additive manufacturing of wood and lignocellulosic filled composites. Polymers 12(9). https ://doi.org/10.3390/polym12092115

Rosenthal M, Henneberger C, Gutkes A, Bues CT (2018) Liquid Deposition Modeling: a promising approach for 3D printing of wood. Eur J Wood Prod 76(2):797-799. https://doi.org/10.1007/s0010 7-017-1274-8

Publisher's Note Springer Nature remains neutral with regard to jurisdictional claims in published maps and institutional affiliations. 